\title{
The Effect of Learning on Bursting
}

\author{
Jan Stegenga*, Joost Le Feber, Enrico Marani, and Wim L. C. Rutten, Senior Member, IEEE
}

\begin{abstract}
We have studied the effect that learning a new stimulus-response (SR) relationship had within a neuronal network cultured on a multielectrode array. For training, we applied repetitive focal electrical stimulation delivered at a low rate $(<1 /$ s). Stimulation was withdrawn when a desired SR success ratio was achieved. It has been shown elsewhere, and we verified that this training algorithm, named conditional repetitive stimulation (CRS), can be used to strengthen an initially weak SR. So far, it remained unclear what the role of the rest of the network during learning was. We therefore studied the effect of CRS on spontaneously occurring network bursts. To this end, we made profiles of the firing rates within network bursts. We have earlier shown that these profiles change shape on a time base of several hours during spontaneous development. We show here that profiles of summed activity, called burst profiles, changed shape at an increased rate during CRS. This suggests that the whole network was involved in making the changes necessary to incorporate the desired SR relationship. However, a local (path-specific) component to learning was also found by analyzing profiles of single-electrode-activity phase profiles. Phase profiles that were not part of the SR relationship changed far less during CRS than the phase profiles of the electrodes that were part of the SR relationship. Finally, the manner in which phase profiles changed shape varied and could not be linked to the $S R$ relationship.
\end{abstract}

Index Terms-Cultured neuronal networks, electrical stimulation, multielectrode arrays (MEAs), synaptic plasticity.

\section{INTRODUCTION}

A LTERING the processing of information by (electrical) stimulation in cultured neuronal networks on multielectrode arrays (MEAs) is something that many groups have struggled with, or still do [1]-[7]. This may have as much to do with the inherent difficulty of observing changes as with the multitude of culturing and maintenance methods. Apart from inducing changes, where some activity-related measure is changed by stimulation, we ultimately want to be able to predict and control these changes.

The conditional repetitive stimulation (CRS) protocol, introduced by Shahaf and Marom [8], strengthened one electrode's responsiveness to stimuli at another electrode. This kind of control has not been reported by other algorithms applicable to dissociated cultures. Shahaf and Marom only reported on the difference between series of test stimuli before and after the experiment. Li et al. [9] recently used the CRS algorithm to

Manuscript received June 16, 2008; revised September 1, 2008. First published October 31, 2008; current version published May 6, 2009. Asterisk indicates corresponding author.

*J. Stegenga is with the Institute of Biomedical Technology (BMTI), Department of Electrical Engineering, Mathematics and Computer Science (EEMCS), Biomedical Signals and Systems Group (BSS), University of Twente, Enschede $7500 \mathrm{AE}$, The Netherlands (e-mail: j.stegenga@utwente.nl).

J. Le Feber, E. Marani, and W. L. C. Rutten are with the Institute of Biomedical Technology (BMTI), University of Twente, Enschede 7500 AE, The Netherlands.

Digital Object Identifier 10.1109/TBME.2008.2006856 study the contribution of various glutamate receptors on learning dynamics. They found that AMPA receptors were primarily involved in altering temporal patterns, while NMDA receptors were mostly responsible for altering spatial patterns.

We investigated the changes made within the network in order to accommodate the desired stimulus-response (SR) relationship. These could be local (i.e., pathway-specific), involving only the stimulus and evaluation sites, or global, involving many sites. Purely pathway-specific interactions would make the CRS expandable to more SR relationships. We evaluated network activity by calculating the relationship of each electrode to spontaneously elicited network-wide synchronous events, i.e., bursts. These are short $(50 \mathrm{~ms}-1 \mathrm{~s})$ intervals in which most neurons fire several action potentials, which develop spontaneously in cultured networks and persist throughout the cultures' lifetime [10]-[15]. Since action potentials within (single neuron) bursts are transmitted reliably by otherwise unreliable synaptic pathways [16], the network connectivity is reflected well by the spatiotemporal structure of network bursts. We previously analyzed the natural development of bursts in vitro through profiles of the instantaneous firing rate during bursts [17]. This revealed that both burst profiles (based on activity summed across all electrodes) and phase profiles (electrode-specific) changed with an average time base of several hours. Furthermore, we showed that phase profiles changed at different rates, indicating a dependency on local interactions as well as global interactions.

The incorporation of a new SR relationship is probably the result of interactions between many neurons. The latency at which the SR is observed (up to $100 \mathrm{~ms}$ ) and the fact that many neurons are active at that time (a network burst is triggered by the stimulus) support this. When training is successful, we expect that it is the triggered network burst that has changed such that the desired SR relationship is incorporated. Analysis of bursts may reveal the spatial extent of the changes, if any, caused by CRS training.

\section{Materials AND MethodS}

\section{A. Culturing}

Cortices were taken from newborn Wistar rats. The cells were dissociated mechanically by trituration and chemically by trypsin. The centers of MEAs were coated with polyethylimine, after which a drop of medium was applied. The plating concentration was 1 million cells $/ \mathrm{ml}$, which resulted in a monolayer of cells with a density of $\sim 2500$ cells $/ \mathrm{mm}^{2}$ after two days in vitro. Cultures were stored in an incubator with $5 \% \mathrm{CO}_{2}$ to air mixture, and near $100 \%$ humidity at $37{ }^{\circ} \mathrm{C}$. Cultures were refreshed twice a week with R12 culturing medium [18], supplemented with $5 \%$ bovine serum. The same medium was also used during measurements. R12 is a minimum essential medium for culturing neurons, but with added bovine serum the glial cells 
multiplied at a low rate. Pilot experiments indicate that the cultures were more active when bovine serum was added, probably due to better functioning of the glial cells (data not shown). Culture ages ranged from 10 to 55 days in vitro (DIV), with nine out of a total of 12 experiments performed in the mature phase $(\geq 21$ DIV).

\section{B. Setup}

We use MEAs and measurement setup (1060BC preamplifier and STG1002 stimulus generator) manufactured by MultiChannel Systems, Reutlingen, Germany. The MEAs had 60 electrodes that were either 10 or $30 \mu \mathrm{m}$ in diameter, which were spaced either 100 or $200 \mu \mathrm{m}$ apart, respectively. During measurements, the temperature at the bottom of the MEA was kept at $36^{\circ} \mathrm{C}$, and a humidified and heated stream of air with $5 \%$ $\mathrm{CO}_{2}$ was blown over the setup. Cultures were sealed with a semipermeable membrane.

Measurements were controlled entirely by custom LabView (National Instruments, Austin, TX) programs. Spikes were detected whenever the signal crossed a threshold of 5.5 times the rms noise level and were validated online using a scheme adopted from Wagenaar et al. [19]. In this algorithm, a putative spike is accepted when it is the highest amplitude of either polarity in a window of $\pm 1 \mathrm{~ms}$. In addition, no spikes of the same polarity and having an amplitude $\geq 50 \%$ as the putative spike may exist in the same window. No spike sorting was attempted in this study for several reasons. First, the shape of the extracellular potential may change within network bursts. Second, both the CRS algorithm and the profile analysis method work well with signals from neural assemblies. Finally, the selection criteria for the evaluation electrodes excluded multiunit activity.

\section{CRS Algorithm}

The CRS algorithm was introduced by Shahaf and Marom [8]. It was their observation that stimuli applied at a low rate $(0.3-$ $1 \mathrm{~Hz}$, such that burst could be triggered) to a single electrode caused changes in functional connectivity within the network. The responses to the stimuli were monitored, and when a desired response appeared often enough (responsiveness), the stimuli were stopped. A single response was defined as one or more spikes detected on a single evaluation electrode within a certain window after stimulus onset (e.g., from 40 to $60 \mathrm{~ms}$ ). The responsiveness was calculated as the moving average of the last ten responses. The target responsiveness was set at two out of ten, where the initial responsiveness was about one out of ten. When the target responsiveness was achieved, or after $10 \mathrm{~min}$ of stimulation, the stimuli were withdrawn. This was repeated with 5-min intervals. The number of stimuli required to elicit the desired response was used as a measure for how well the new SR relationship was learned. Our procedure was as follows.

1) Whenever possible, a long period $(>6 \mathrm{~h})$ of spontaneous activity was measured before test stimuli were applied. These spontaneous measurements were used to assess normal developmental changes in the absence of stimuli.

2) The culture was probed with stimuli of varying amplitude (4-20 $\mu \mathrm{A}$, biphasic, $200 \mu \mathrm{s} /$ phase, negative phase

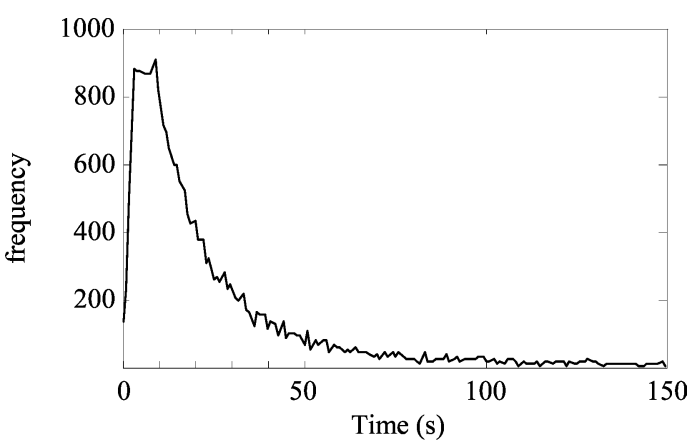

Fig. 1. Distribution of interburst intervals. Data from spontaneous measurements before and after all CRS experiments were used. The distribution peaks near $6 \mathrm{~s}$, while the average IBI is $25 \mathrm{~s}$. The bin size used is $1 \mathrm{~s}$.

first) at a rate of $0.2 / \mathrm{s}$. The poststimulus histograms of array-wide activity (awPSTH) were visually examined to select a small set of stimulus electrodes.

3) A second series of test stimuli was applied to each candidate stimulus electrode. At this point, the stimulation rate was adjusted such that the ratio of evoked bursts to stimuli was maximized. The stimulation rate was usually 1.5-3 times higher than the typical spontaneous interburst interval (IBI). The IBI distribution is given in Fig. 2. Interstimulus intervals consequently varied between 1.5 and $5 \mathrm{~s}$. The minimum stimulation rate was set at $0.1 / \mathrm{s}$ and the ratio at which bursts were evoked had to be above 0.8 in order to continue.

4) A third series of test stimuli was applied at the chosen stimulus electrode and chosen rate. PSTHs of each electrode were calculated, and the evaluation electrode and response window had to be chosen. The criterion was that the area of PSTH within the response window was 0.1 . Multiple spikes can be triggered by a single stimulus, so this method may have overestimated the responsiveness. However, for electrodes with this low responsiveness, it was not problematic. Unlike Shahaf and Marom, who used a fixed evaluation window (i.e., 40-60 ms), we varied the response window such that the peak in the poststimulus histogram of the evaluation electrode was within it. Window widths varied between 20 and $50 \mathrm{~ms}$, with the earliest starting at $10 \mathrm{~ms}$ after stimulus and the latest ending at $100 \mathrm{~ms}$. This was done because: a) the network-wide response varied between cultures and between ages and b) it required connection strength rather than latency to change.

5) Between the test series and the start of CRS training, an hour of spontaneous activity was measured (Fig. 1).

6) Start of the CRS experiment: A learning experiment was stopped when a stable fast responsiveness was reached, or when the network-wide response (i.e., number of spikes within $300 \mathrm{~ms}$ after stimulus) to stimuli became lower than $80 \%$ of its initial value. The initial value was calculated by taking the average of the first five iterations of the CRS algorithm and the exact criterion was applied offline. Since a (permanent) degradation in network response resulted in CRS not reaching its goal within $10 \mathrm{~min}$, these experiments (four in total) were ended by the experimenter a few 


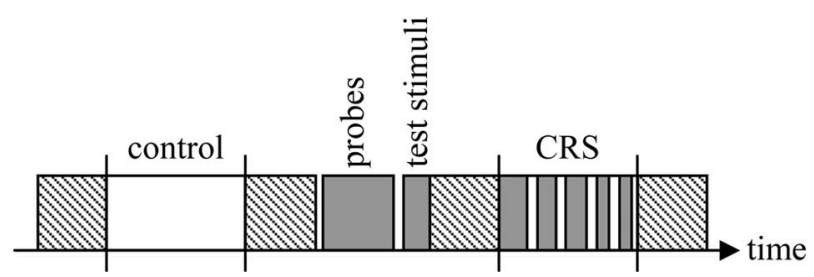

Fig. 2. Time line of a measurement. Control experiments of equal length as CRS experiments were used for comparing rates of change during spontaneous development with those during CRS. Control and CRS experiments were preceded and followed by $1-\mathrm{h}$ measurements of spontaneous activity to compare the effects of CRS with spontaneous development (hatched). Control and CRS experiments were less than $8 \mathrm{~h}$ apart. Preparation for a CRS experiment consisted of selecting a stimulus electrode (probes) and selecting an evaluation electrode/window (test stimuli). Shaded boxes designate periods of stimulation.

iterations after degradation had set in. Testing for stability of the awPSTH during CRS was not attempted because of variability as iterations of the protocol may comprise as few as three stimuli. Shahaf and Marom have already stated that the network response (i.e., triggered network burst) should be stable throughout the experiment, but did not quantify this.

7) After the learning experiment was concluded, spontaneous activity was once again recorded.

Next to the learning curve, which consists of the number of stimuli required to reach the desired responsiveness, we calculated: 1) the average network (i.e., on all electrodes) response, calculated by counting the average number of spikes in $500 \mathrm{~ms}$ following a stimulus; 2) the average network response within the desired window; and 3) the average evaluation electrode response within the desired window. All responses were normalized to the value in the first iteration (CRS) or to the average of the first 5 min (spontaneous) to enable comparison between experiments.

\section{Profile Analysis}

Profiles of the within-burst firing rate were calculated from spontaneous activity measured before, during, and after CRS experiments (Fig. 1). The procedure was described earlier [17]. Examples of burst and phase profiles can be found in Fig. 3. The analysis consists of four steps: burst detection, burst profile calculation, phase profile calculation, and profile averaging. First, bursts were detected by dividing the summed activity in 100-ms bins and applying a threshold. The threshold was set at two times the number of electrodes that showed action potential activity (i.e., an average firing rate $>0.1$ spikes/s). Second, burst profiles were calculated by convolving the spike times (summed over all electrodes) in a burst with a Gaussian. The standard deviations (SDs) were between 5 and $15 \mathrm{~ms}$, depending on the activity. Smooth burst profiles near the main peak were necessary for further processing. The profile was calculated from 200 $\mathrm{ms}$ before the main peak in the burst profile to $600 \mathrm{~ms}$ after the peak. Third, 60 phase profiles were calculated by convolving the spike times within the above-defined window of each individual electrode with a Gaussian with the same SD as used for the burst profile. Fourth, we aligned burst profiles to their main
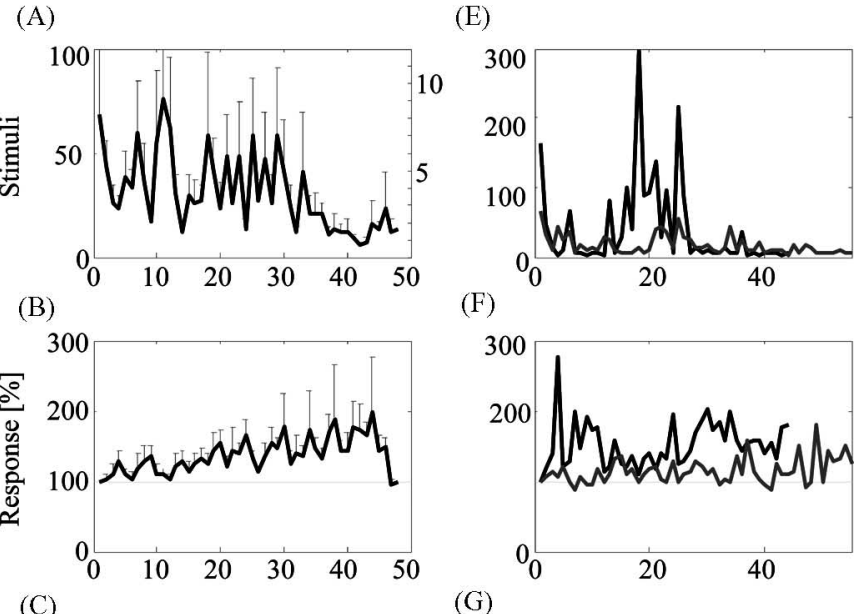

(F)
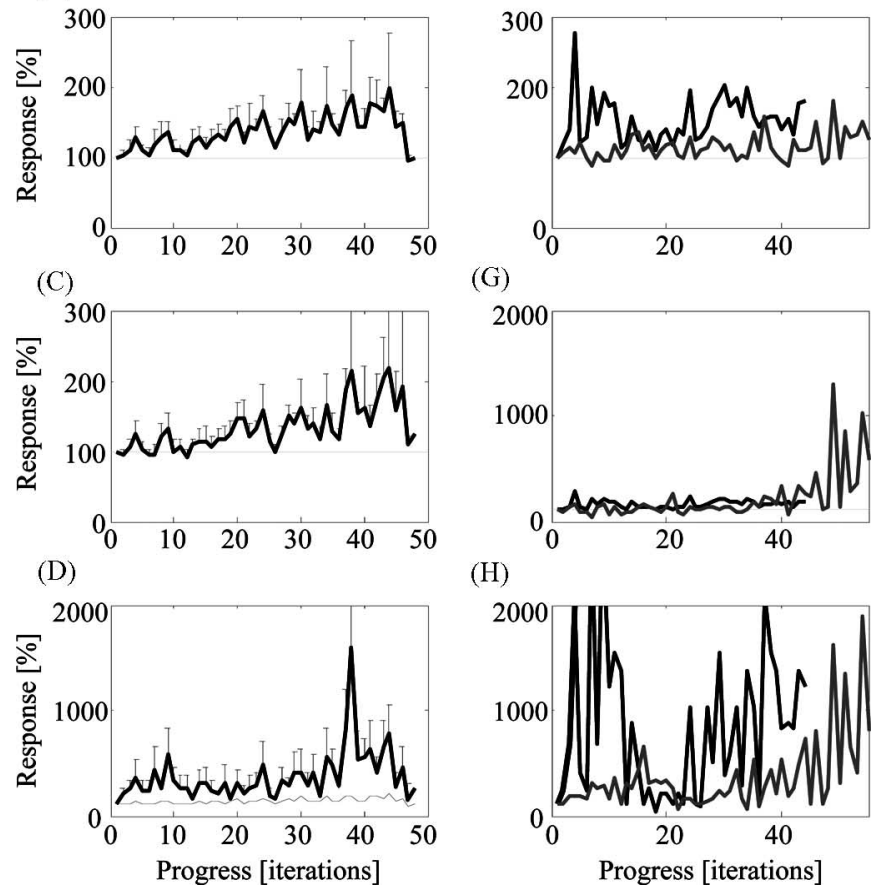

(G)
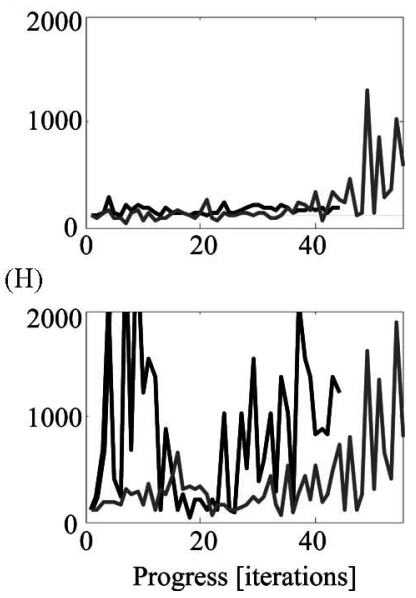

Fig. 3. (A) Average learning curve. The number of stimuli required to elicit the desired response decreased. Slope: $-0.749 \pm 0.157$ per iteration (mean \pm standard error); Kendall's $\tau:-0.450(p<0.001)$. Curves show mean + standard error. The right $y$-axis and the gray line show the number of experiments that the curves are based on. (B) Development of the array-wide response. Slope: $0.010 \pm 0.002$; Kendall's $\tau: 0.466$ ( $p<0.001)$. Individual curves were scaled to the response in the first iteration before averaging. (C) Development of the array-wide response within evaluation windows. Evaluation windows differ between experiments. Slope: $0.017 \pm 0.003$; Kendall's $\tau: 0.546(p<0.001)$. (D) Normalized response on the evaluation electrode. This response consists of spikes on the evaluation electrode within the evaluation window, again normalized to the value in the first iteration. Slope: $0.068 \pm 0.023 ;$ Kendall's $\tau: 0.282$ $(p=0.004)$. In gray, the normalized array-wide response is reproduced. (E) and (H) Same curves for two example experiments.

peak and made 5-min averages. This was done because there may be very few spikes elicited by some electrodes in a single burst. Averaging reduced the variability of phase profiles. An alternative would be to use Gaussians with a large SD, but doing so would lose timing information. We treated the resulting average profiles as if they were profiles from individual bursts.

We used the rms value of the difference between two profiles to quantify change. We normalized the rms value to the area of the profile (i.e., the number of spikes)

$$
\begin{aligned}
D_{i} & =\frac{\operatorname{rms}\left(p_{i}-p_{\text {ref }}\right)}{\operatorname{rms}\left(p_{\text {ref }}\right)} \\
\operatorname{rms}(x) & =\operatorname{sqrt}\left(\frac{1}{N} \sum_{n=1}^{N} x_{n}^{2}\right)
\end{aligned}
$$




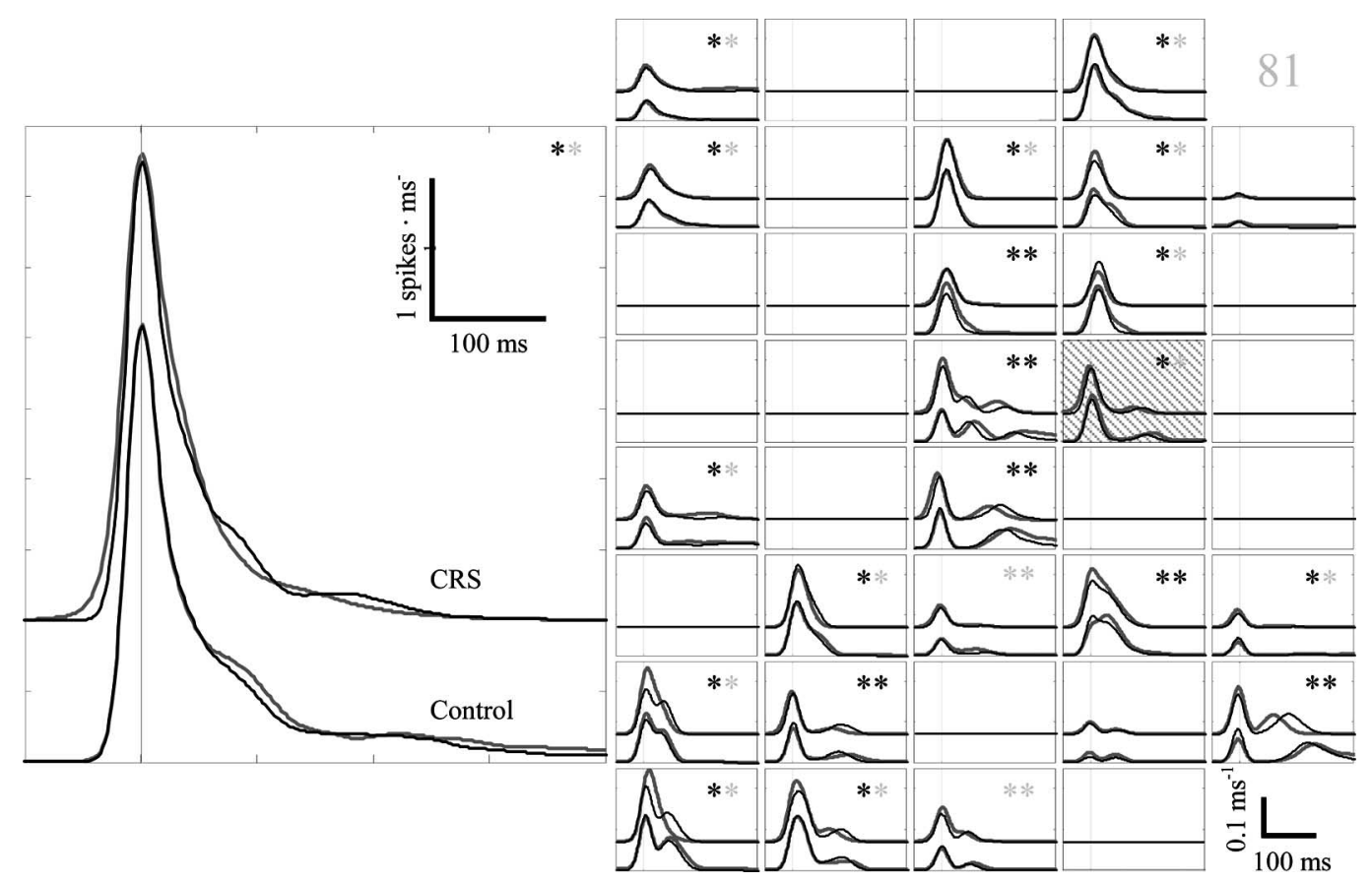

Fig. 4. (Left panel) Example of changes in burst profiles immediately before and after CRS (top two traces, black equals before, gray after). The lower two traces are immediately before and after an equal period of spontaneous development. All curves are 45-min averages. (Right panels) Phase profiles of the most active part of the MEA in the same experiment, shown in the same layout as the electrodes. The hatched plot is at the location of the stimulus electrode (i.e., 74); evaluation was at electrode 73. Significantly changed profiles are denoted by black asterisks, left for CRS and right for control.

where $D_{i}$ is the normalized distance between profile $p_{i}$ and a reference profile $p_{\text {ref }}$, and each profile consists of $N$ samples. The width of the profiles was $600 \mathrm{~ms}$, containing 601 data points. This distance measure is sensitive to changes in profile shape and to changes in overall firing rate and was applied to both burst and phase profiles. The rate of change of $D$ during CRS experiments was calculated using the 5 min of spontaneous activity between iterations. The reference profile that we used within CRS was the average profile during the first 5-min pause. For control measurements, we used the average profile during the first $5 \mathrm{~min}$ as a reference. When one of the spontaneous measurements before the CRS experiment was of equal length (or longer) than the CRS experiment $(N=4)$, the change in profiles during the experiment was directly compared to the change over an equal length of time of spontaneous activity. Significance of changes was determined using two-sided Student's $t$-tests. The significance of slopes was tested using the following data:

$$
a_{i}=\frac{y_{i}-b}{x_{i}}
$$

where $b$ is the intercept calculated by normal linear regression and $\left(x_{i}, y_{i}\right)$ are the original data points.

\section{Results}

\section{A. Learning Experiments}

Fig. 3(A) shows the average learning curve of 12 experiments performed on nine different cultures. The averaged curve has a negative slope, indicating successful learning. Individual learning curves [Fig. 3(E)] could be quite erratic and were qualitatively described by fast initial learning, followed by a relapse starting somewhere between the 10th and 20th iteration before a stable (low) value was reached. During the experiment, the array-wide number of spikes elicited by stimulation increased slightly, as seen in Fig. 3(B) and (C). Since the experiments were of various lengths, the last two data points are averages of only two experiments, which may account for the discrepancy between these values and the general trend. In contrast, the number of spikes elicited on the evaluation electrode within the response window [Fig. 3(D)] increased fast during the first three iterations, stabilized around a value of 2.2, and then increased once more. The examples in Fig. 3(D)-(F) show that during the relapse, in these examples between iterations 13 and 28, the response on the evaluation electrode momentarily reached prelearning levels, while the array-wide response did not change during this period.

\section{B. Profiles}

Profiles of spontaneous bursts before and after learning were compared with measurements separated by the same amount of time as the learning protocol (control). These were taken either before or after learning. Fig. 4 shows an example of a learning experiment and controls taken before learning. During the control measurement, the burst profile remained unchanged, and nine out of 25 phase profiles were changed. During learning, 20 out of 25 phase profiles changed significantly, and this resulted in the significant change of the burst profile as well. The 

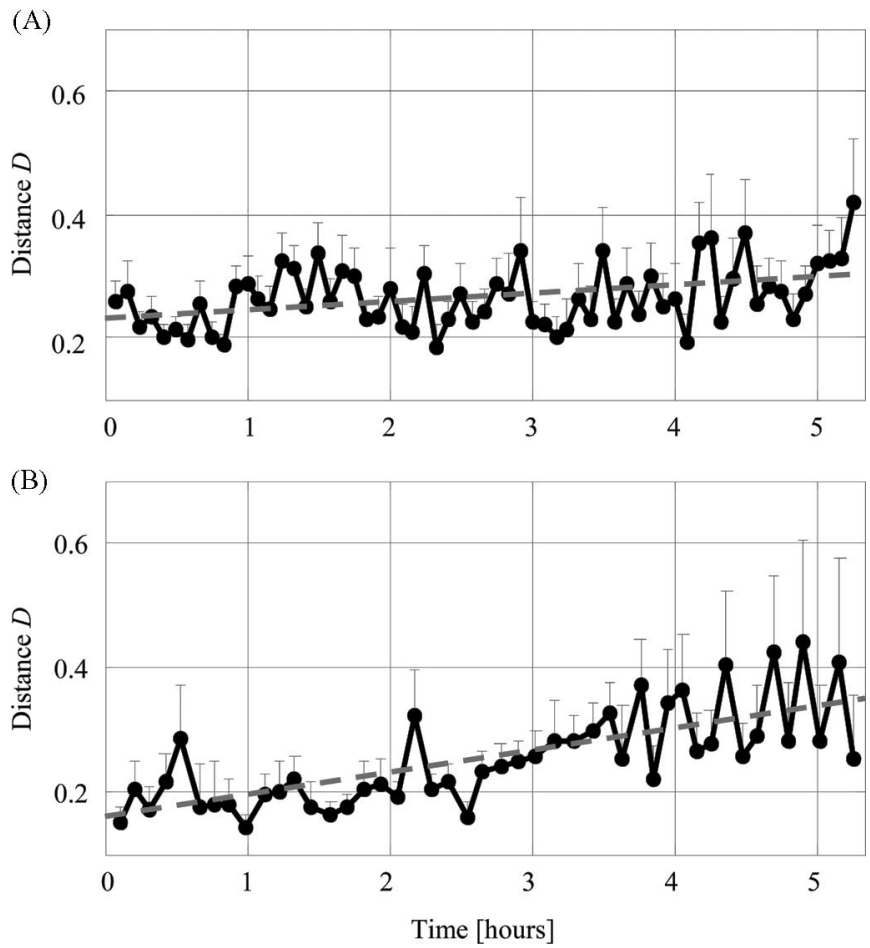

Fig. 5. Development of distance $D$ of change of burst profiles. (A) Development of $D$ of burst profiles in control experiments $(N=4)$. Slope: 0.012 $\pm 0.004 / \mathrm{h}$; Kendall's $\tau: 0.220(p=0.011)$. Graphs show mean + standard error. (B) Development of $D$ of burst profiles during CRS $(N=12)$. The first 5 -min pause, which is after the first stimulation round, was taken as a reference. Slope: $0.036 \pm 0.005 / \mathrm{h}$ (mean \pm standard error); Kendall's $\tau: 0.585(p<0.001)$. The difference between the two slopes was significant $(p=0.0015)$.

right panels in Fig. 4 also illustrate the complexity that phase profiles can have, most notably a second or even third phase of firing. In the four experiments in which controls were possible, all burst profiles changed during CRS compared to two spontaneous burst profiles changes (Student's $t$-test; $p \leq 0.01$ ). For phase profiles, $57 \%$ changed during CRS, while $46 \%$ changed spontaneously. On average, there were 13 active electrodes per culture in these experiments.

The distances between burst profiles are shown in Fig. 5 . Variability in the burst profiles is represented by a baseline (the average value in the first hour) of about 0.2 . The sensitivity of the profile method to profile changes is given by the baseline. Of greater importance is the presence of a positive slope, which indicates a progressive change from the initial profile. An increase in distance can be observed in the CRS experiments after $150 \mathrm{~min}$, which roughly corresponds to the 22nd iteration. Even though the standard errors are high, a positive slope $(0.036 / \mathrm{h})$ can be seen during the CRS protocol. During spontaneous measurements, a positive slope of only $0.012 / \mathrm{h}$ is less visible. Calculations of Kendall's $\tau$ correlation coefficient show a clear positive correlation during CRS and a smaller correlation during spontaneous development.

The distances between phase profiles are shown in Fig. 6 . Again, a baseline can be seen around 0.34 during spontaneous measurements (top panel) and 0.41 during CRS (lower panels). These baselines are higher than their burst profile counterparts
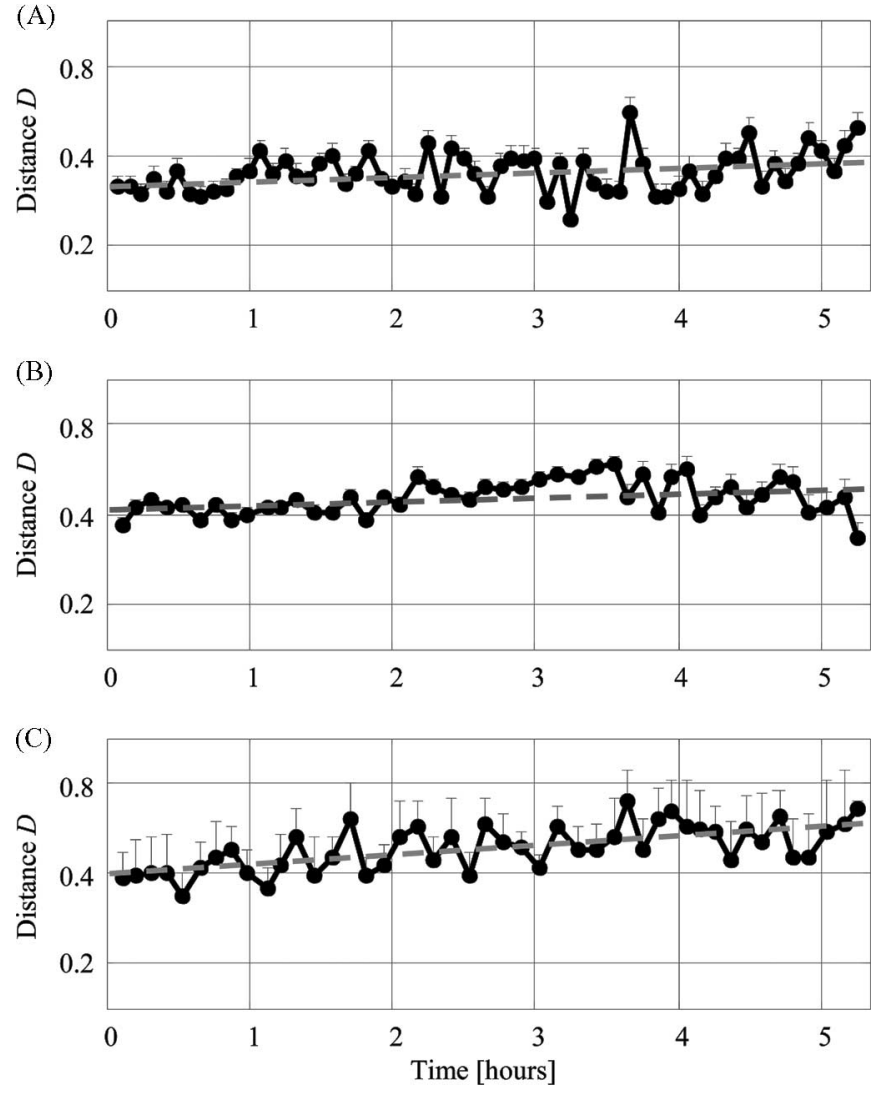

Fig. 6. Development of distance $D$ of phase profiles. (A) Development of phase profiles during control $(N=4)$. Slope: $0.009 \pm 0.004 / \mathrm{h}$; Kendall's $\tau: 0.203(p=0.019)$. (B) Development of phase profiles on all electrodes except those used for evaluation during CRS $(N=12)$. Slope: $0.008 \pm 0.003 / \mathrm{h}$; Kendall's $\tau: 0.283(p=0.005)$. (C) Change of the phase profiles of evaluation electrodes during CRS $(N=12)$. Slope: $0.021 \pm 0.004 / \mathrm{h}$; Kendall's $\tau: 0.473$ $(p<0.001)$. Graphs show mean + standard error. The difference between curves A and B was not significant $(p=0.6161)$, while curve C differed significantly from both A and B ( $p=0.0203 ; p=0.0069)$.

in Fig. 5, indicating the higher variability of phase profiles, and thus, also a decreased sensitivity to changes. In this case, the calculated slopes for spontaneous development and electrodes not used by CRS are comparable, while the slope for electrodes used for evaluation show a much higher slope. Kendall's $\tau$ correlation coefficients show that phase profiles on electrodes not used by CRS are more strongly correlated (with time) than phase profiles during spontaneous development. Phase profiles on electrodes used for CRS have the highest correlation coefficient $(0.021 / \mathrm{h})$. Next to the rate of change, a change was also seen between profiles immediately before and after CRS (Fig. 7). The average duration of CRS, $5 \mathrm{~h}$, was longer than the typical time base of spontaneous change, which is several hours [17]. Therefore, a change was observed during control measurements in which no stimulation was applied. However, Fig. 6 shows that the rate of change of burst profiles during CRS exceeded the spontaneous rate. The phase profiles showed the same, in that all phase profiles showed a small but significant increase in the rate of change during CRS. The increase was considerably larger on evaluation electrodes than on other electrodes, indicating a pathway-specific component in training using CRS. 


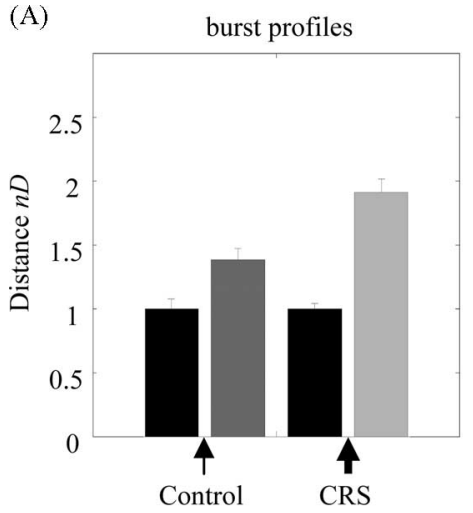

(B) phase profiles

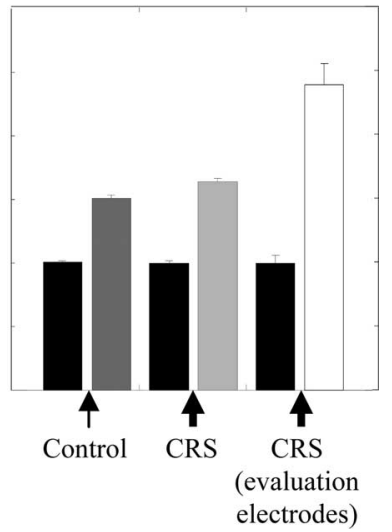

Fig. 7. (A) Changes between burst profiles immediately before and immediately after control (small arrow) and CRS experiments (bold arrows). Distances $(D)$ were renormalized to the average distance in the "before" groups (black bars). Bars show mean + standard error of mean. (B) Changes between phase profiles. All means were statistically different (all $p<0.005$, Student's $t$-test).
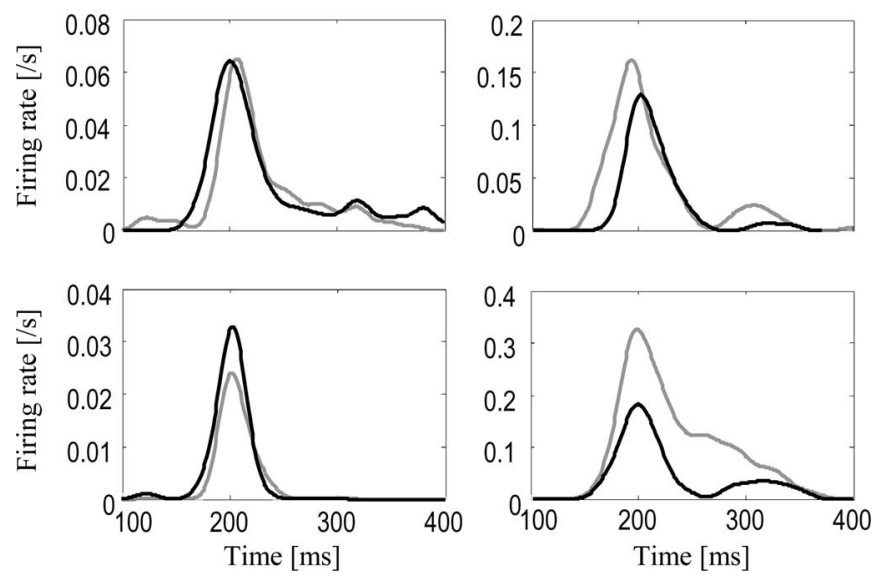

Fig. 8. Four examples illustrate the types of changes between phase profiles at the start of CRS (black) and at the end (gray). We observed shifts of the main peak in three cases, an increase in peak firing rate in four cases, a decrease in peak firing rate in six cases, and an increase in second phase amplitude in five cases.

How did the phase profiles change then? Phase profiles from four CRS experiments are shown in Fig. 8. A decrease in peak firing rate was observed in most cases, but also shifts in the position of the main peak and in some cases an increasing second phase were observed. The decrease in firing rate of phase profiles was often accompanied with a decrease in firing rate of burst profiles. We have observed such changes during spontaneous activity as well, albeit it is rare to observe an increase in second phase spontaneously. It should be noted that out of the eight cases in which the stimulation electrode was also spontaneously active, there were six cases in which phase profiles on the evaluation electrode increased in size relative to phase profiles on the stimulation electrode. Therefore, a decrease in size of a phase profile on evaluation electrode may reflect an overall decrease in activity and does not exclude strengthening of the relationship between stimulus and evaluation electrode.

\section{DISCUSSION}

\section{A. CRS Protocol}

The selection procedure before starting the learning experiment itself was critical for the success of the experiment. For instance, the CRS paradigm was designed to enhance connectivity strength of initially low-strength connections, but not to a very high responsiveness (from $1 / 10$ to $2 / 10$ ). Using CRS to increase responsiveness from $\sim 3 / 20$ to $3 / 12$, or from $1 / 10$ to $3 / 10$, was never successful (data not shown). This limits the general usefulness of CRS. Next to cultures that did not have a stable network response at interstimulus intervals smaller than $10 \mathrm{~s}$, we also had to discontinue experiments (4 out of 12) because the network-wide response to stimuli (i.e., awPSTH) deteriorated during the course of an experiment. Such deterioration in awPSTH was seen when spontaneous bursts started occurring in between stimuli, which also increased the variability of the response. Whether all this was due to changes in the entire network or locally at the stimulus site is subject of further investigation. When observing $80 \%$ criterion, the awPSTH area steadily increased [Fig. 3(B)]. However, the (relative) increase of PSTH area on the evaluation electrode [Fig. 3(D)] was far greater.

In a number (i.e., 14) of cases, we found that the criterion was reached very quickly in the first iterations of CRS, despite our efforts to select an evaluation electrode with a low responsiveness. Changes that may have occurred during the test stimuli or during the spontaneous activity that separated test stimuli from the start of CRS may be the cause of this. These experiments were aborted, as there was no further learning possible. Shahaf and Marom did not report on the number of cultures that did not fulfill the boundary conditions, but in our investigation, only nine out of 22 (otherwise active and easy to stimulate) cultures met the prerequisites [8]. Initial learning curves (first ten iterations) resembled the examples given by Shahaf and Marom. Further iterations of the CRS protocol often showed a period with a relapse in learning, something that was not reported earlier. Consequently, a stable low value was reached much later. We found it necessary to adjust the evaluation window to a value that corresponded to a latency at which the activity was elicited. The larger differences in latency that we found may be due to the fact that we used MEAs with two different electrode spacings or due to a larger spread in culture age (10-55 DIV). However, more subtle differences in preparation and maintenance cannot be excluded. Despite the erratic learning rates, we found that the cultures that met all the prerequisites were trainable. One possibility to reduce variability in responses, which may result in smoother learning curves, is to create a pool of evaluation neurons [20]. However, it is not known what impact this may have on learning.

\section{B. Profile Change}

The rate at which both burst and phase profiles (of evaluation electrodes) changed during CRS stimulation was higher than during spontaneous development. However, phase profiles on electrodes that were not used during CRS changed 
at a rate comparable to spontaneous development (judged by slope). Since the burst profiles did change, and a burst profile is essentially a summation of phase profiles, this result was unexpected. However, the changes in phase profiles are normalized to the area of the profile (i.e., the activity of the electrode). On the other hand, the influence of electrodes on the burst profile is activity-dependent. Therefore, the results indicate that highly active sites changed more rapidly. Also, given the relatively large rate of change of burst profiles, it is likely that a number of phase profiles changed in the same general direction. Such convergent changes are commonly seen during spontaneous development only on a larger time scale. From this point of view, the stimulation accelerated, but did not alter the normal development.

In contradiction to this view, we observed that phase profile on electrodes used for evaluation during CRS changed at a very high rate. This result is surprising because the phase profiles on evaluation electrodes were always small (low spiking activity), and large phase profiles generally had a higher rate of change. This cannot be explained merely by a speeding up of normal development. Instead, by withdrawing stimuli when the desired response is achieved, the changes occurring on the evaluation electrodes were "cultivated," while changes on other electrodes may go back and forth and negate each other. The CRS algorithm thus relies on accelerating changes by means of stimuli (i.e., induction exploratory behavior [8]), and preserving these changes on the evaluation electrodes.

The coupling between phase profiles on evaluation electrodes and SR relationships is complex. A phase profile shows one electrode's contribution to network collective bursts, while an SR relationship only involves two electrodes. It is through the fact that stimulation elicits bursts, that the two are coupled. The complexity of this coupling is probably also the reason why no correlation between the stimulation-evaluation electrode pair and the changes in phase profiles could be found. Comparing phase profiles immediately before CRS to phase profiles immediately after CRS showed a small increase in the number of phase profile changes and also an increase in distance $D$.

The results suggest that the whole network was involved during training in a lesser degree and that stimulation and evaluation electrodes were particularly involved. Assuming that a change in phase profile (site-specific) is related to the change in responsiveness (pathway-specific), it is not clear whether changes in evaluated phase profile depend on the changes in other phase profiles or even burst profiles. Since only one SR relationship is controlled, it may simply be that the rest of the network incorporates this change in a way that requires the least modifications. Conversely, it may also be that changes throughout the whole of network are driving the SR relationship toward a higher efficacy. Whatever the case, expanding the CRS algorithm to multiple SR relationships will not be straightforward, partly due to global network interactions and partly due to the selection criteria for trainable SR relationships.

\section{ACKNOWLEDGMENT}

The authors would like to thank R. Wiertz for his work on the preparation and maintenance of cultures.

\section{REFERENCES}

[1] M. Chiappalone, P. Massobrio, and S. Martinoia, "Network plasticity in cortical assemblies," Eur. J. Neurosci., vol. 28, pp. 221-237, 2008.

[2] Y. Jimbo, T. Tateno, and H. P. C. Robinson, "Simultaneous induction of pathway specific potentiation and depression in networks of cortical neurons," Biophys. J., vol. 76, pp. 670-678, 1999.

[3] R. Madhavan, Z. C. Chao, and S. M. Potter, "Plasticity of recurring spatiotemporal activity patterns in cortical networks," Phys. Biol., vol. 4, pp. 181-193, 2007.

[4] E. Maeda, Y. Kuroda, H. P. Robinson, and A. Kawana, "Modification of parallel activity elicited by propagating bursts in developing networks of rat cortical neurones," Eur. J. Neurosci., vol. 10, pp. 488-496, 1998.

[5] S. Marom and D. Eytan, "Learning in ex-vivo developing networks of cortical neurons," Prog. Brain Res., vol. 147, pp. 189-199, 2005.

[6] M. E. Ruaro, P. Bonifazi, and V. Torre, "Toward the neurocomputer: Image processing and pattern recognition with neuronal cultures," IEEE Trans. Biomed. Eng., vol. 52, no. 3, pp. 371-383, Mar. 2005.

[7] D. A. Wagenaar, J. Pine, and S. M. Potter, "Searching for plasticity in dissociated cortical cultures on multi-electrode arrays," J. Negative Results Biomed., vol. 5, pp. 16-35, 2006.

[8] G. Shahaf and S. Marom, "Learning in networks of cortical neurons," $J$. Neurosci., vol. 21, pp. 8782-8788, 2001.

[9] Y. Li, W. Zhou, X. Li, S. Zeng, M. Liu, and Q. Luo, "Characterization of synchronized bursts in cultured hippocampal neuronal networks with learning training on microelectrode arrays," Biosens. Bioelectron., vol. 22, pp. 2976-2982, 2007.

[10] M. Chiappalone, M. Bove, A. Vato, M. Tedesco, and S. Martinoia, "Dissociated cortical networks show spontaneously correlated activity patterns during in vitro development," Brain Res., vol. 1093, pp. 41-53, 2006.

[11] H. Kamioka, E. Maeda, Y. Jimbo, H. P. Robinson, and A. Kawana, "Spontaneous periodic synchronized bursting during formation of mature patterns of connections in cortical cultures," Neurosci. Lett., vol. 206, pp. 109-112, 1996

[12] E. Maeda, H. P. Robinson, and A. Kawana, "The mechanisms of generation and propagation of synchronized bursting in developing networks of cortical neurons," J. Neurosci., vol. 15, pp. 6834-6845, 1995.

[13] J. Van Pelt, M. A. Corner, P. S. Wolters, W. L. Rutten, and G. J. Ramakers, "Long-term stability and developmental changes in spontaneous network burst firing patterns in dissociated rat cerebral cortex cell cultures on multielectrode arrays," Neurosci. Lett., vol. 361, pp. 86-89, 2004.

[14] J. van Pelt, P. S. Wolters, M. A. Corner, W. L. Rutten, and G. J. Ramakers, "Long-term characterization of firing dynamics of spontaneous bursts in cultured neural networks," IEEE Trans. Biomed. Eng., vol. 51, no. 11, pp. 2051-2062, Nov. 2004.

[15] D. A. Wagenaar, J. Pine, and S. M. Potter, "An extremely rich repertoire of bursting patterns during the development of cortical cultures," $B M C$ Neurosci., vol. 7, no. 11, 2006

[16] J. E. Lisman, "Bursts as a unit of neural information: Making unreliable synapses reliable," Trends Neurosci., vol. 20, pp. 38-43, 1997.

[17] J. Stegenga, J. Le Feber, E. Marani, and W. C. Rutten, "Analysis of cultured neuronal networks using intraburst firing characteristics," IEEE Trans. Biomed. Eng., vol. 55, no. 4, pp. 1382-1390, Apr. 2008.

[18] H. J. Romijn, F. van Huizen, and P. S. Wolters, "Towards an improved serum-free, chemically defined medium for long-term culturing of cerebral cortex tissue," Neurosci. Biobehav. Rev., vol. 8, pp. 301-334, 1984.

[19] D. A. Wagenaar, T. B. DeMarse, and S. M. Potter, "MeaBench: A toolset for multi-electrode data acquisition and online analysis," in Proc. 2nd Int. IEEE EMBS Conf. Neural Eng., Arlington, VA, 2005, pp. 518-521.

[20] P. Bonifazi, M. E. Ruaro, and V. Torre, "Statistical properties of information processing in neuronal networks," Eur. J. Neurosci., vol. 22, pp. 2953-2964, 2005.

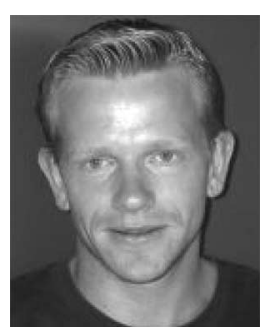

Jan Stegenga was born in Wijckel, The Netherlands, on April 19, 1979. He received the M.Sc. degree in 2004 in electrical engineering from the University of Twente, Enschede, The Netherlands, and the Ph.D. degree in memory and learning in cultured neuronal networks, in 2009.

His current interests include rhythmic activity and information processing in the basal ganglia. 


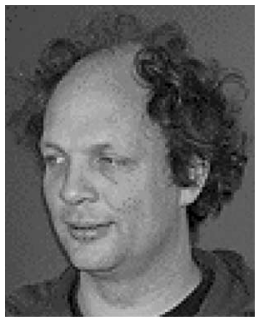

Joost Le Feber was born in Veldhoven, The Netherlands, in 1967. He received the M.Sc. degree in electrical engineering from Delft University of Technology, Delft, The Netherlands, in 1991, and the $\mathrm{Ph} . \mathrm{D}$. degree in quantitative models that relate autonomic nerve activity to mechanical variables in the lower urinary tract from Erasmus University, Rotterdam, The Netherlands.

He was a Postdoctoral Fellow at Groningen University, where he worked in the field of artificial intelligence for four years. He is currently with the University of Twente, Enschede, The Netherlands. His current research interests include learning and memory, cultured neuronal networks, and computational neuroscience.

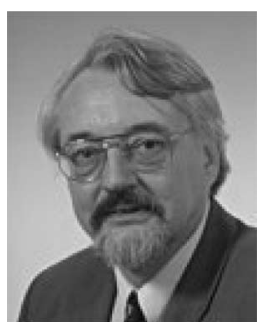

Enrico Marani was born in 1946. He received the $\mathrm{Ph} . \mathrm{D}$. degree in the structure and function of the cerebellum from Leiden University, Leiden, The Netherlands, in 1982.

He was trained as a Neuroanatomist at Leiden University. In 1986, he became the Head of the Neuroregulation Group at Leiden University Medical Center. Since 1990, he has been studying the regeneration of the central nervous system. He is currently with the Institute of Biomedical Technology (BMTI), University of Twente, Enschede, The Netherlands. His current research interests include the application of neurotechnology in medicine.

Dr. Marani is an Editor of Advances in Anatomy, Embryology, and Cell Biology, and a Co-Editor of BioMedical Reviews.

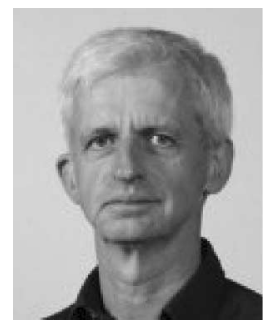

Wim L. C. Rutten (M'96-SM'04) was born in 1950. He received the Ph.D. degree in solid-state physics from Leiden University, Leiden, The Netherlands, in 1979

He was a Physicist-Audiologist in the Hearing Science and Electrophysiology Department, Leiden University Hospital, until 1985 . Then he joined the Biomedical Engineering Group, University of Twente, Enschede, The Netherlands, where he is the Chair of Neurotechnology at the Faculty of Electrical Engineering, Mathematics, and Computer Science and at the Institute for BioMedical Technology (BMTI), where he is also the Leader of the Neurotechnology and Cellular Engineering Program. His current research interests include live learning neural networks, development of (cultured) peripheral neuroprostheses, and EEG-based brain-computer interfaces. 